\title{
Effect of prone and supine treatment positions for postoperative treatment of rectal cancer on target dose coverage and small bowel sparing using intensity-modulated radiation therapy
}

\author{
Yongqiang Yang ${ }^{1,2,3 \#}$, Shang Cai ${ }^{1,2,3 \#}$, Tianshu Zhao ${ }^{1,2,3 \#}$, Qiliang Peng ${ }^{1,2,3}$, Jianjun Qian $^{1,2,3}$, Ye Tian ${ }^{1,2,3}$ \\ ${ }^{1}$ Department of Radiotherapy \& Oncology, The Second Affiliated Hospital of Soochow University, Suzhou 215004, China; ${ }^{2}$ Institute of Radiotherapy \\ \& Oncology, Soochow University, Suzhou 215004, China; ${ }^{3}$ Suzhou Key Laboratory for Radiation Oncology, Suzhou 215004, China \\ Contributions: (I) Conception and design: Y Tian, J Qian; (II) Administrative support: Y Tian, J Qian; (III) Provision of study materials or patients: Q \\ Peng, T Zhao; (IV) Collection and assembly of data: S Cai, T Zhao; (V) Data analysis and interpretation: Y Yang, S Cai, T Zhao; (VI) Manuscript \\ writing: All authors; (VII) Final approval of manuscript: All authors. \\ "These authors contributed equally to this work. \\ Correspondence to: Ye Tian; Jianjun Qian. Department of Radiotherapy \& Oncology, The Second Affiliated Hospital of Soochow University, San \\ Xiang Road No. 1055, Suzhou 215004, China. Email: dryetian@126.com; qianjianjun0628@126.com.
}

\begin{abstract}
Background: To evaluate the influence of patient positioning on target dose coverage and dose distribution to the small bowel in intensity-modulated radiation therapy (IMRT) for rectal cancer patients.

Methods: Twenty-four consecutive rectal cancer patients undergoing postoperative radiation were selected and set up in either a prone or supine position. All patients received computed tomography (CT) scans before and during treatment (1-4 weeks). The CT images were defined as plan, $1 \mathrm{~W}, 2 \mathrm{~W}, 3 \mathrm{~W}$, or $4 \mathrm{~W}$. The plan, $1 \mathrm{~W}, 2 \mathrm{~W}, 3 \mathrm{~W}$ and $4 \mathrm{~W}$ CT images were fused. The clinical target volume (CTV) and planning target volume (PTV) delineated on the plan CT were copied onto the 1-4W CT. The treatment plans based on the plan CT were also copied onto the $1-4 \mathrm{~W}$ CT. The target dose coverage rate was assessed. The couchposition data of the linear accelerator were acquired.
\end{abstract}

Results: Failure rates of the CTV and PTV target dose coverage were higher in the prone group than in the supine group $(18.60 \%$ vs. $0 \%$ and $69.76 \%$ vs. $53.65 \%)$ during the treatment. The total couch-position deviation for the prone group $(1.23 \pm 0.76 \mathrm{~cm})$ was significantly greater than that of the supine group $(0.28 \pm$ $0.18 \mathrm{~cm} ; \mathrm{P}=0.001)$. Compared with the supine group, the prone group had significantly less irradiated small bowel volume at V5 $(\mathrm{P}=0.003)$ and V10 $(\mathrm{P}=0.004)$.

Conclusions: The supine position maintained better target dose coverage and setup reproducibility in rectal cancer patients treated with IMRT. The prone position combined with the belly board can reduce the dose received by the small bowel.

Keywords: Rectal cancer; prone; supine; intensity-modulated radiation therapy (IMRT)

Submitted Aug 16, 2019. Accepted for publication Nov 11, 2019.

doi: $10.21037 /$ tcr.2019.11.33

View this article at: http://dx.doi.org/10.21037/tcr.2019.11.33

\section{Introduction}

Patient positioning in rectal cancer radiotherapy should be determined based on setup reproducibility and the position that yields the lowest radiation dose to the organs at risk (OARs), especially the small bowel. Supine and prone positions are the most common treatment positions for rectal cancer patients undergoing radiotherapy. Previous studies have demonstrated that setup reproducibility for the different positions is an important influential factor of therapeutic outcomes (1-3). The supine setup is generally 
Table 1 Patient demographic and clinical features $(n=24)$

\begin{tabular}{lcc}
\hline Characteristics & Prone $(\mathrm{n}=12)(\%)$ & Supine $(\mathrm{n}=12)(\%)$ \\
\hline Age (years), median [range] & $55[35-76]$ & $53[38-71]$ \\
Patients with stoma & $6(50.0)$ & $5(41.7)$ \\
Surgical procedure & & \\
Mile's & $7(58.3)$ & $5(41.7)$ \\
Dixon & $5(41.7)$ & $7(58.3)$ \\
Gender & & \\
Male & $7(58.3)$ & $6(50.0)$ \\
Female & $5(41.7)$ & $6(50.0)$ \\
Pathologic T stage & & $2(16.7)$ \\
T2 & $3(25.0)$ & $8(66.7)$ \\
T3 & $5(41.7)$ & $2(16.7)$ \\
T4 & $3(25.0)$ & \\
Pathologic N stage & & $5(25.0)$ \\
N0 & $3(25.0)$ & $5(41.7)$ \\
N1 & $5(41.7)$ & $4(33.3)$ \\
N2 & $73-9]$ & \\
Distance from anal verge & & \\
(cm), median [range] & & \\
\hline
\end{tabular}

associated with more stability during irradiation, easier setup, and more patient comfort, while the prone setup, especially on a belly board, is associated with a reduced dose to the small bowel. Due to the steep dose distribution gradient in intensity-modulated radiation therapy (IMRT), target dose coverage should be closely monitored. Although setup errors are accounted for in the margin expansion from clinical target volume (CTV) to planning target volume (PTV), the margin is also affected by factors such as radiotherapeutic technique, treatment position, and the immobilization device; thus, the margin must be confirmed by practical outcomes. This study compared the effect of the supine and prone positions on target dose coverage, thereby providing a reference for the optimal position in rectal cancer IMRT.

\section{Methods}

\section{General clinical information}

This study was conducted as a retrospective review. The recruitment period was from January to June 2018. Twentyfour patients with rectal cancer undergoing postoperative IMRT in our department were included and divided into the supine $(\mathrm{n}=12)$ and prone $(\mathrm{n}=12)$ groups. Table 1 summarizes the patients' characteristics.

\section{Immobilization and computer tomography (CT) scans}

All patients were moved into the CT simulation (Siemens Emotion Duo) gantry head first. The supine group was immobilized using a carbon fiber base plate (CIVCO Medical Solutions, Orange City, Iowa, USA) and thermoplastic mask (Orfit, Wijnegem, Belgium). The prone group was immobilized using the belly board device (CIVCO Medical Solutions, Orange City, Iowa, USA). The CT simulation scans used a 5 -mm slice thickness and spacing, ranging from the third lumbar vertebra to the lower anal verge. Before the CT scans, patients were instructed to fill their bladders and take the Gastrografin solution $(500 \mathrm{~mL})$ to better visualize the small bowel. CT simulation images were imported into the Pinnacle 9.0 treatment planning system (Philips Radiation Oncology, Fitchburg, Wisconsin, USA) and named "plan CT". Next, the target volume was delineated, and the treatment was planned. After verification, the treatment plan was delivered to the patients on the Synergy accelerator (Elekta, Elekta Oncology Systems, Crawley, UK). CT scans were repeated under the same conditions on Fridays of the 1st-4th weeks of treatment and named $1 \mathrm{~W}, 2 \mathrm{~W}, 3 \mathrm{~W}$, and $4 \mathrm{~W}$, respectively.

\section{Small bowel delineation}

The small bowel delineation method was described by the Radiation Therapy Oncology Group (RTOG) and Banerjee et al. $(4,5)$. Based on the contrast effect, the small bowel was delineated in every CT slice by contouring the outer surface with the upper border $1 \mathrm{~cm}$ above the PTV and the lower border at the end of the small bowel. The colon was excluded. All delineations were completed by one radiotherapy physician, with a window width of 600 and window level of 40 .

\section{Definitions of target volume and treatment planning}

The target volume delineation and dose prescription were in accordance with the RTOG and international expert consensus guide $(6,7)$. The CTV included the mesorectal 
area, presacral space, and internal iliac and obturator lymph node areas. The ischial-rectal fossa and the external iliac lymph node area were also included in some patients. The PTV was an expansion of the CTV: a $1 \mathrm{~cm}$ expansion in both the cranial and caudal directions and a $0.5 \mathrm{~cm}$ expansion to the left, right, front, and back. The prescribed dose was 50 Gy for 25 fractions. The Pinnacle 9.0 planning system was applied to set up the 7-field IMRT treatment plan based on the plan CT. The treatment plan used a $6 \mathrm{MV}$ $\mathrm{X}$-ray, cc convolution calculation, $0.3 \mathrm{~cm}$ computing grid, Elekta Synergy accelerator, and 40 MLC (multi-leaf collimator) pairs. The organs at risk (OAR) dose constraint was V15 $<275$ cc for the small bowel, V40 $<50 \%$ for the bladder, and V50 $<5 \%$ for the bilateral femoral heads (5). The target dose coverage requirements were that $100 \%$ of the prescribed dose should include $>95 \%$ of the PTV. The maximum PTV dose (Dmax) was <54 Gy. The 1-4W CT images were fused with the plan CT images. The two treatment plans based on the plan CT were copied to the $1-4$ W CT.

\section{Target dose coverage rates and failure rates of the two groups in different positions}

The CTV and PTV target dose coverage rates for 50 Gy were acquired from the dose-volume histograms (DVH) based on the plan CT and the 1-4W CT. The failure rate for the target dose coverage was defined as the ratio of the number of times that the target dose coverage was less than $95 \%$ to the total number of measurements.

\section{Evaluation of the small bowel dose volume}

The volume of the irradiated small bowel was defined as the absolute volume of V5-V50 (the volume of small bowel irradiated with 5-50 Gy in 5 Gy increments). Each patient's small bowel volume (or the irradiated volume) was the mean volume of all CT slices. The small bowel volume (irradiated volume) of all patients over the entire therapy period was the median of each patient's mean volumes.

\section{Prediction of the small bowel NTCP}

The built-in Lyman-Kutcher-Burman (LKB) calculation module in Pinnacle 9.0 was applied to predict chronic complications of the small bowel and was referred to as the chronic normal tissue complication probability $\left(\mathrm{NTCP}_{\mathrm{C}}\right)$ (8-10). The parameters $\mathrm{n}$ (volume factor), $\mathrm{m}$ (slope of dose reaction curve), and TD50 (mean doses with a 50\% probability of complications) were set as $0.15,0.16$, and 55 Gy, respectively (11). Complications were defined as a small bowel obstruction, perforation, or fistula. The logistic formula, NTCP $=\left(1+(\mathrm{V} 50 / \mathrm{V})^{\mathrm{k}}\right)^{-1}$, was used to calculate the acute toxicity of V15 (i.e., $\mathrm{NTCP}_{\mathrm{A}}$ ). V50 and k were set as $130 \mathrm{cc}$ and 1.1, respectively (12). The method for obtaining NTCP statistical data for each or all patients was the same as that used to obtain the small bowel volume (or irradiated volume).

\section{Accelerator couch-position data}

Self-developed software was used in the Elekta MOSAIQ network (Elekta Oncology Systems, Crawley, UK) to acquire the couch-position data for each treatment cycle on the Synergy accelerator. Three directions (X, Y, and Z) were included: $+X$ and $-X$ represented the patient's left and right, $+Y$ and $-Y$ represented the cranial-caudal direction (the beam and target ends of the accelerator), and $+Z$ and $-Z$ represented the ventral-dorsal (rising and falling directions of the accelerator couch). At the end of the radiotherapy, a total of 25 datasets were acquired for each patient in the 3 directions. The primary setup, which was completed by a team composed of a radiation oncologist, a radiation physicist and a technologist, was similar to the position at CT simulation due to the short time interval; thus, the couch position of the first therapy course was used as the baseline. The subsequent 24 couch-position datasets were compared with the baseline to generate the couch-position deviation for each therapy course and the mean deviation for the 25 fractions $(\Delta x, \Delta y, \Delta z)$. The total couch-position deviation $(\mathrm{S}, \mathrm{cm})$ was calculated as:

$$
S=\sqrt{\Delta x^{2}+\Delta y^{2}+\Delta z^{2}}
$$

\section{Statistical methods}

SPSS 22.0 statistical software was used. A paired $t$-test was performed to compare the difference between the two groups. Pearson's analysis was applied to analyze the correlation. $\mathrm{P}<0.05$ was considered statistically significant.

\section{Results}

\section{Image data and treatment planning}

One hundred nine CT-scan image sets were obtained 

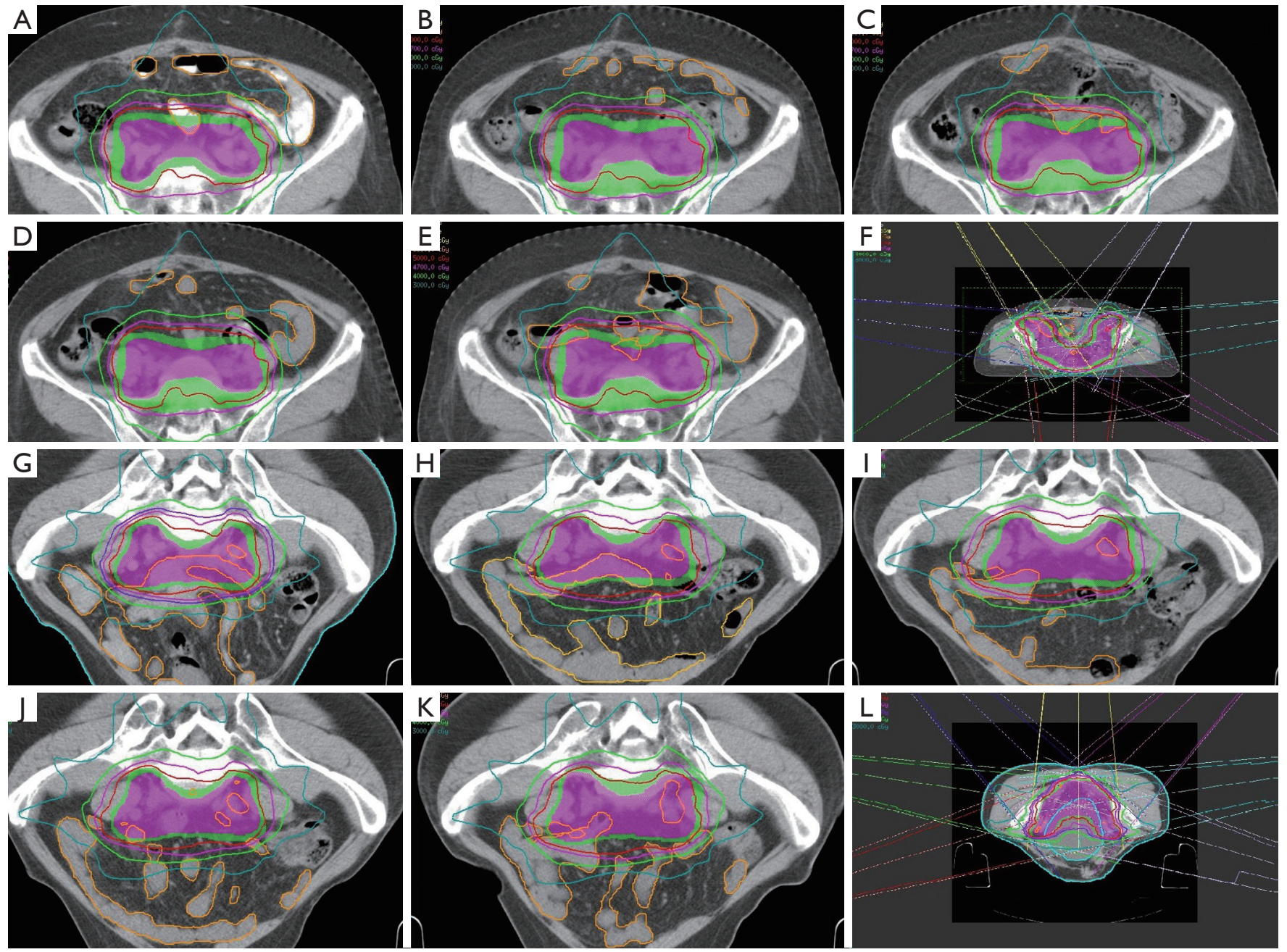

Figure 1 Treatment planning and target volume for one supine and one prone patient. (A,B,C,D,E,F) Supine patient [(A) plan CT, (B,C,D,E) 1-4W CT, (F) field setup]. (G,H,IJ,K) Prone patient [(G) plan CT, (B,C,D,E) 1-4 W CT, (L) field setup]. The green, purple, and orange contours represent the PTV, CTV, and small bowel volume, respectively. The innermost and outermost isodose lines indicate 5,000 and 3,000 cGy, respectively. CTV, clinical target volume; PTV, planning target volume.

from the 24 patients, among which 24 sets were plan, $2 \mathrm{~W}$, and $3 \mathrm{~W} ; 14$ sets were $1 \mathrm{~W}$; and 23 sets were $4 \mathrm{~W}$. The 24 treatment plans based on the plan CT were copied to the 1-4W CT for a total 109 treatment plan sets. All treatment plans met the target dose coverage and OAR constraint requirements. Figure 1 shows the target volume and treatment planning of a supine patient and a prone patient.

\section{Couch-position deviation}

The mean couch-position deviations in the $\mathrm{X}, \mathrm{Y}$, and $\mathrm{Z}$ directions were $0.07 \pm 0.36,0.02 \pm 0.92$, and $0.08 \pm 0.36 \mathrm{~cm}$, respectively. The total couch-position deviation in the prone group $(1.23 \pm 0.76 \mathrm{~cm})$ was significantly higher than that in the supine group $(0.28 \pm 0.18 \mathrm{~cm} ; \mathrm{P}=0.001)$. The greatest deviations were seen in the $\mathrm{Y}$ (cranial-caudal) and $\mathrm{Z}$ (ventral-dorsal) directions $(\mathrm{P}=0.003$ and $\mathrm{P}=0.003$, respectively; Table 1).

\section{Target dose coverage}

Table 2 shows the patients' target dose coverage rates. The two groups' CTV target dose coverage rates (supine $v s$. prone) were $98.69 \% \pm 0.86 \%$ vs. $97.51 \% \pm 2.20 \%$ 
Table 2 Target dose coverage and couch-position deviations of the 24 rectal cancer patients

\begin{tabular}{|c|c|c|c|c|c|}
\hline Variable & Total patients & Supine patients & Prone patients & $t$ & $\mathrm{P}$ \\
\hline PTV target coverage (\%) & $94.28 \pm 2.42$ & $95.07 \pm 2.16$ & $93.49 \pm 2.50$ & 1.68 & 0.120 \\
\hline Couch-position deviation X (cm) & $0.07 \pm 0.36$ & $0.04 \pm 0.25$ & $0.09 \pm 0.46$ & 1.66 & 0.123 \\
\hline Couch-position deviation Y (cm) & $0.02 \pm 0.92$ & $0.06 \pm 0.21$ & $0.02 \pm 1.32$ & -3.66 & 0.003 \\
\hline Total couch-position deviation S (cm) & $0.74 \pm 0.73$ & $0.28 \pm 0.18$ & $1.23 \pm 0.76$ & -4.15 & 0.001 \\
\hline
\end{tabular}

$t$ and $\mathrm{P}$ are the comparisons between the supine and prone groups. The couch positions were compared with the absolute couch-position deviation. CTV, clinical target volume; PTV, planning target volume.

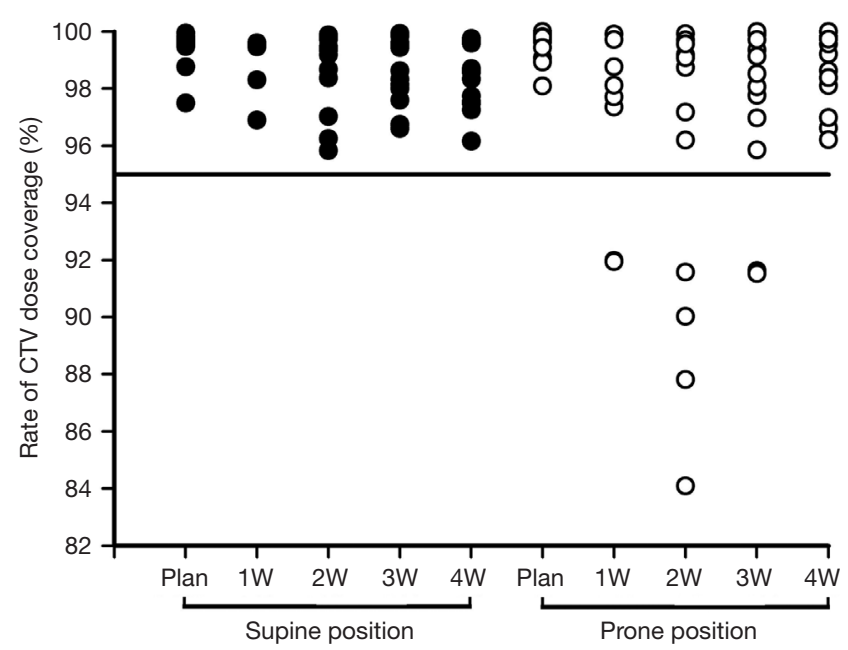

Figure $2 \mathrm{CTV}$-prescribed dose coverage rates for 24 rectal cancer patients ( $\bullet$ supine patients; oprone patients; straight horizontal line: prescribed dose coverage rate of $95 \%)$. CTV, clinical target volume.

$(\mathrm{P}=0.148)$, while the PTV target dose coverage rates were $95.07 \% \pm 2.16 \%$ vs. $93.49 \% \pm 2.50 \%(\mathrm{P}=0.120)$. Figures 2 and 3 demonstrate the CTV and PTV target dose coverage rates. The CTV dose coverage rates of the 12 supine patients were all above $95 \%$. For five patients (41.67\%) in the prone group, the CTV target dose coverage rate dropped below $95 \%$ eight times; the lowest was $84.09 \%$. The prone group had higher CTV target coverage failure rates than the supine group (18.60\% vs. $0 \%)$. Eight patients $(66.66 \%)$ in the supine group and $10(83.33 \%)$ in the prone group experienced more than one drop in PTV target dose coverage to below $95 \%$; the lowest was $77.10 \%$. The prone group had higher PTV target coverage failure rates than the supine group (69.76\% vs. $53.65 \%)$. Figure 1 shows the treatment planning and target volumes of 2 patients. Figure $1 A, B, C, D, E$ shows that the supine patient's position was changed in the cranial-caudal direction. Figure $1 G$, $H, I, \mathcal{F}, K$ shows that the prone patient was moved in both the cranial-caudal direction and the left-right direction. Figure 4 shows the correlational analysis of the target dose coverage rate and total couch-position deviation, indicating a significant correlation $(\mathrm{R}=-0.683, \mathrm{P}=0.000)$.

\section{Small bowel dose volume and NTCP}

Table 3 shows the small bowel dose volumes and NTCP for the 24 patients. Most of the dose volumes ranging from V5V50 (excluding V50) were higher in the supine group than in the prone group, and the differences at V5 and V10 were statistically significant $(\mathrm{P}=0.003$ and 0.004 , respectively). The supine group showed greater acute and chronic small bowel toxicity $\left(\mathrm{NTCP}_{\mathrm{A}}, \mathrm{NTCP}_{\mathrm{C}}\right)$ than the prone group: $\mathrm{NTCP}_{\mathrm{A}} 58.95 \% \pm 6.70 \%$ vs. $57.77 \% \pm 8.65 \%(\mathrm{P}=0.248)$; $\mathrm{NTCP}_{\mathrm{C}} 4.78 \% \pm 2.59 \%$ vs. $2.70 \% \pm 1.67 \%(\mathrm{P}=0.041)$.

\section{Discussion}

Sufficient target dose coverage should be prioritized when selecting patient positioning for rectal cancer IMRT. Setup reproducibility is usually affected by the patient's physical condition, comfort, and immobilization devices. Poor setup reproducibility reduces target dose coverage. This study evaluated the target dose coverage in rectal cancer patients in the supine and prone positions. The doses received and the NTCP of the two patient groups were also evaluated. This study provides a reference for choosing optimal patient 
Table 3 Small bowel dose volume and NTCP of 24 rectal cancer patients undergoing IMRT

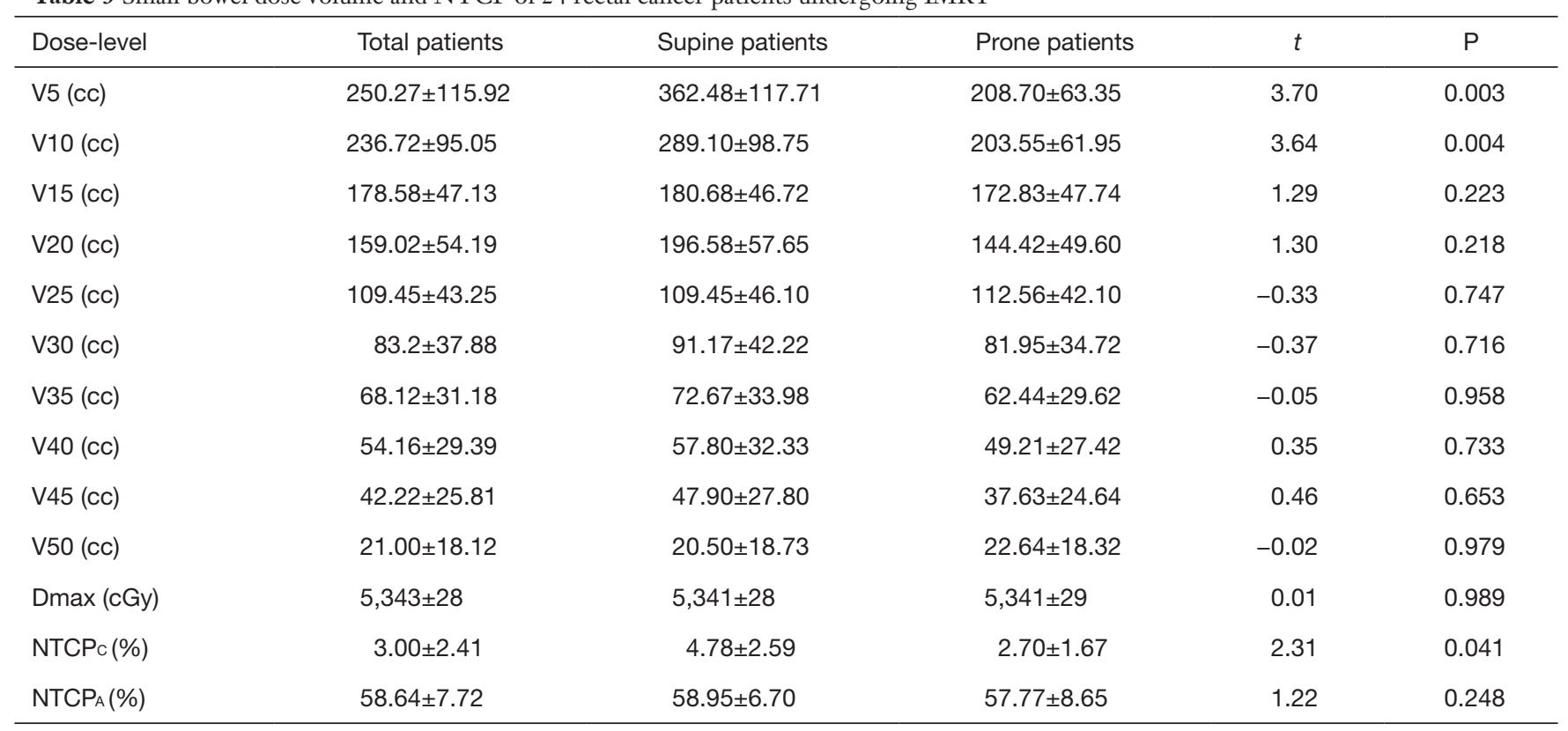

$t$ and $\mathrm{P}$ are the comparisons between the supine and prone groups. NTCPC and NTCPA are the chronic and acute normal tissue complication probabilities, respectively. IMRT, intensity-modulated radiation therapy; NTCPc, chronic normal tissue complication probability; NTCPA, acute normal tissue complication probability.

positioning in rectal cancer IMRT.

Compared with the supine position, the prone position reduces the patient's comfort and immobilization. In addition, the immobilizing thermoplastic mask cannot be used in combination with the belly board in most prone positions. The couch-position deviation was used in this study to describe the setup reproducibility. The setup reproducibility is affected by the interconnection of the center of the tumor, skin marks, immobilization device marks, and accelerator couch, for which the couch-position deviation is a very good indicator $(13,14)$. Our results showed that the prone group had greater total couchposition deviation $(\mathrm{S})$ than the supine group $(\mathrm{P}=0.001)$; the deviation was apparent in the cranial-caudal $(\mathrm{P}=0.003)$ and ventral-dorsal $(\mathrm{P}=0.003)$ directions. Similarly, previous literature has also reported that the prone position decreases setup reproducibility, especially in the cranial-caudal and ventral-dorsal directions, and the belly board increases setup error (15-18).

The prone position also reduced the CTV and PTV target dose coverage rates, although the differences were not statistically significant. Five patients $(41.67 \%)$ in the prone group experienced 8 occurrences of the CTV target dose coverage rate dropping to $<95 \%$ : twice at $1 \mathrm{~W}, 4$ times at $2 \mathrm{~W}$, twice at $3 \mathrm{~W}$, and none at $4 \mathrm{~W}(2 \mathrm{~W}$ was the greater). The lowest CTV target dose coverage rate was only $84.09 \%$ from the same patient who also had the lowest PTV target dose coverage rate of $77.10 \%$ (Figures 2,3). The CTV and PTV target dose coverage failure rates in the prone group were higher than those in the supine group. Further analysis revealed that the target dose coverage rate was correlated with the total couch-position deviation $(\mathrm{R}=-0.683, \mathrm{P}=0.000$; Figure 4), indicating that setup reproducibility can affect the target dose coverage rate, thereby providing references for patient setup with a fixed couch position. Because the geometric position between the PTV and CTV was fixed in this study, their relationship to the setup reproducibility was similar (Figure 4). However, the CTV target dose coverage rate was lower than that of the PTV, which could lead to insufficient dose distribution to target volume or even therapy failures. Therefore, the prone position has problems regarding stable target dose coverage.

Compared with the supine position, the prone position significantly reduced the small bowel dose volumes at V5 and V10 ( $\mathrm{P}=0.003$ and 0.004 respectively) but not at V15-50. Drzymala et al. selected 19 patients undergoing preoperative chemoradiation and performed simulation CT scanning in both the supine and prone (without the belly 


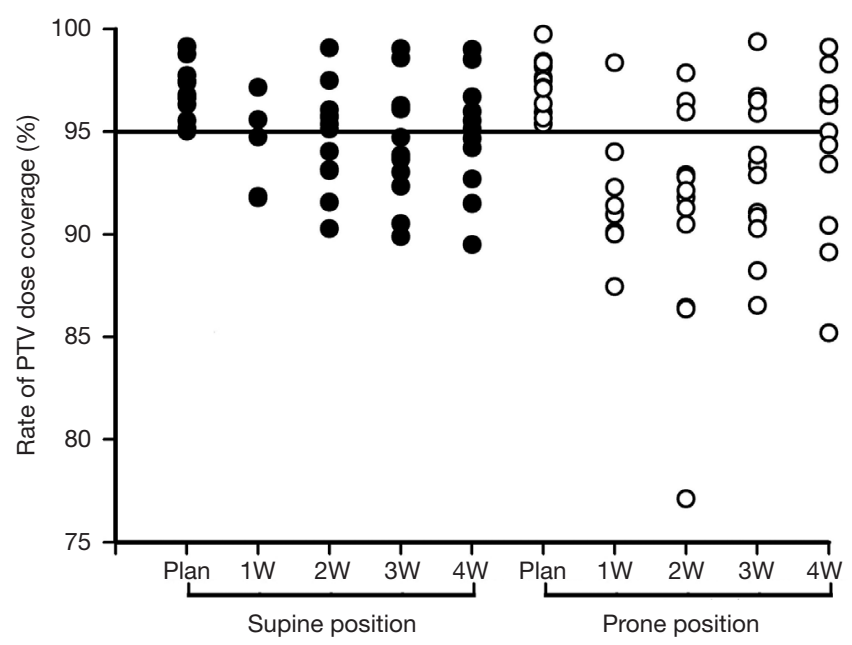

Figure 3 PTV-prescribed dose coverage rates for 24 rectal cancer patients ( $\bullet$ supine patients; oprone patients; straight horizontal line: prescribed dose coverage rate of $95 \%)$. PTV, planning target volume.

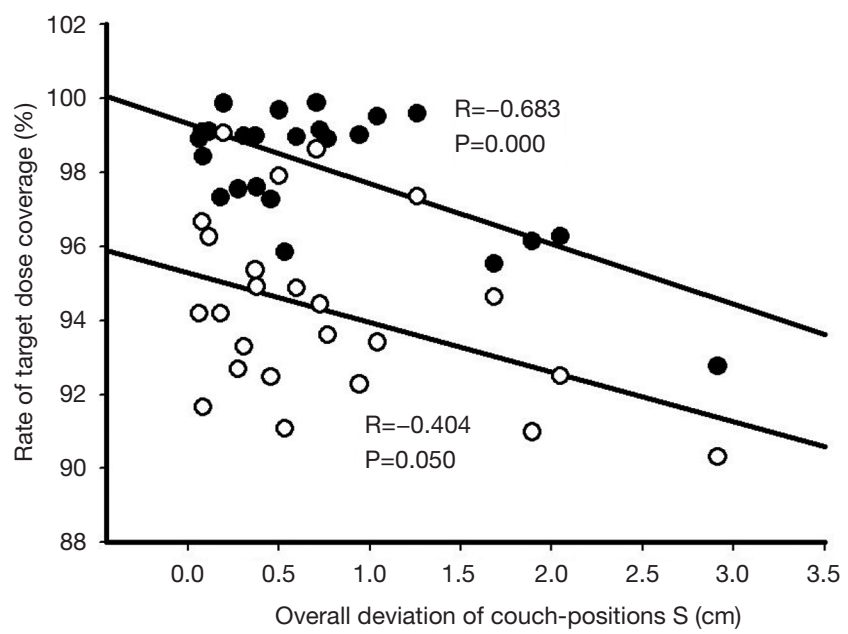

Figure 4 Correlation between the target dose coverage rate and total couch-position deviation for 24 rectal cancer patients $(\bullet \mathrm{CTV}$ prescribed dose coverage rate; $\circ \mathrm{PTV}$-prescribed dose coverage rate). CTV, clinical target volume; PTV, planning target volume.

board) positions, along with three-dimensional conformal planning (19). Their results showed a significantly higher irradiated bowel volume (colon included) at V5 and V10 in the supine position, in the range of V5-45. These authors concluded that the dose received by the bowel did not differ between the two positions and that the supine position could be adopted for patients undergoing preoperative rectal cancer radiotherapy because the setup reproducibility was sound. The present study differed from theirs in that the patients were postoperative; the received dose measurement involved only the small bowel, not the colon; and the prone patients used belly boards. Further NTCP analysis in this study showed that the prone position reduced the chronic toxicity $\left(\mathrm{NTCP}_{\mathrm{C}}\right)$ but did not affect the acute toxicity $\left(\mathrm{NTCP}_{\mathrm{A}}\right)$. Previous reports also demonstrated that the prone position combined with the belly board in IMRT could markedly diminish the received dose to the small bowel, and the greatest reduction in irradiated volume occurred at low dose levels $(1,2,20,21)$.

This study only observed the effect of patient positioning in setup errors and target dose coverage. The CTV was copied from the plan CT to the fused $1-4 \mathrm{~W}$ CT without adaptive corrections; thus, the influence of physiological movements of the bladder and other organs was not considered. However, adaptive corrections to the target volume would have complicated the study and even obscured the effect of setup error in the target dose coverage.

\section{Conclusions}

The supine position could enable more stable target dose coverage in rectal cancer IMRT. The prone position combined with the belly board reduced the setup reproducibility, thereby affecting the target dose coverage. Although the prone position combined with the belly board could reduce the dose distribution to the small bowel, effective measures should be taken to ensure patient setup reproducibility.

\section{Acknowledgments}

We thank American Journal Experts for assisting in the preparation of this manuscript.

Funding: This work was supported, in part, by the Jiangsu Provincial Special Program of Clinical Medical Science (No. BE2018657), Jiangsu Medical Innovation Team (No. CXDT-37), and Medicine Outstanding Leader of Suzhou (No. 62), Scientific Research Program for Young Talents of China National Nuclear Corporation (to S Cai), Jiangsu Commission of Health medical research project (No. H2018115). 


\section{Footnote}

Conflicts of Interest: All authors have completed the ICMJE uniform disclosure form (available at http://dx.doi. org/10.21037/tcr.2019.11.33). The authors have no conflicts of interest to declare.

Ethical Statement: The authors are accountable for all aspects of the work in ensuring that questions related to the accuracy or integrity of any part of the work are appropriately investigated and resolved. The study was conducted in accordance with the Declaration of Helsinki (as revised in 2013). The study was approved by the ethics committee of The Second Affiliated Hospital of Soochow University (No. 2018067). Informed consent was waived due to the retrospective nature of the study.

Open Access Statement: This is an Open Access article distributed in accordance with the Creative Commons Attribution-NonCommercial-NoDerivs 4.0 International License (CC BY-NC-ND 4.0), which permits the noncommercial replication and distribution of the article with the strict proviso that no changes or edits are made and the original work is properly cited (including links to both the formal publication through the relevant DOI and the license). See: https://creativecommons.org/licenses/by-nc$\mathrm{nd} / 4.0 /$.

\section{References}

1. Frøseth TC, Strickert T, Solli KS, et al. A randomized study of the effect of patient positioning on setup reproducibility and dose distribution to organs at risk in radiotherapy of rectal cancer patients. Radiat Oncol 2015;10:217.

2. Koeck J, Kromer K, Lohr F, et al. Small bowel protection in IMRT for rectal cancer : A dosimetric study on supine vs. prone position. Strahlenther Onkol 2017;193:578-88.

3. Kim A, Karotki A, Presutti J, et al. The effect of prone and supine treatment positions for the pre-operative treatment of rectal cancer on organ-at-risk sparing and setup reproducibility using volumetric modulated arc therapy. Radiat Oncol 2017;12:180.

4. Gay HA, Barthold HJ, O'Meara E, et al. Pelvic normal tissue contouring guidelines for radiation therapy: a Radiation Therapy Oncology Group consensus panel atlas. Int J Radiat Oncol Biol Phys 2012;83:e353-e362.

5. Banerjee R, Chakraborty S, Nygren I, et al. Small Bowel
Dose Parameters Predicting Grade $\geq 3$ Acute Toxicity in Rectal Cancer Patients Treated With Neoadjuvant Chemoradiation: An Independent Validation Study Comparing Peritoneal Space Versus Small Bowel Loop Contouring Techniques. Int J Radiat Oncol Biol Phys 2013;85:1225-31.

6. Myerson RJ, Garofalo MC, El Naqa I, et al. Elective clinical target volumes for conformal therapy in anorectal cancer: a radiation therapy oncology group consensus panel contouring atlas. Int J Radiat Oncol Biol Phys 2009;74:824-30.

7. Valentini V, Gambacorta MA, Barbaro B, et al. International consensus guidelines on Clinical Target Volume delineation in rectal cancer. Radiother Oncol 2016;120:195-201.

8. Webb S, Nahum AE. A model for calculating tumor control probability in radiotherapy including the effects of inhomogeneous distributions of dose and clonogenic cell density. Phys Med Biol 1993;38:653-66.

9. Burman C, Kutcher GJ, Emami B, et al. Fitting of normal tissue tolerance data to an analytic function. Int J Radiat Oncol Biol Phys 1991;21:123-35.

10. Lyman JT, Wolbarst AB. Optimization of radiation therapy III: a method of assessing complication probabilities from dose volume histograms. Int J Radiat Oncol Biol Phys 1987;13:103-9.

11. Cella L, Ciscognetti N, Martin G, et al. Preoperative Radiation Treatment for Rectal Cancer: Comparison of Target Coverage and Small Bowel NTCP in Conventional vs. 3D-Conformal Planning. Med Dosim 2009;34:75-81.

12. Tho LM, Glegg M, Paterson J, et al. Acute small bowel toxicity and preoperative chemoradiotherapy for rectal cancer: Investigating dose-volume relationships and role for inverse planning. Int J Radiat Oncol Biol Phys 2006;66:505-13.

13. Engelsman M, Rosenthal SJ, Michaud SL, et al. Intraand interfractional patient motion for a variety of immobilization devices. Med Phys 2005;32:3468-74.

14. Hadley SW, Balter JM, Lam KL. Analysis of couch position tolerance limits to detect mistakes in patient setup. J Appl Clin Med Phys 2009;10:2864.

15. Weber DC, Nouet P, Rouzaud M, et al. Patient positioning in prostate radiotherapy: is prone better than supine? Int J Radiat Oncol Biol Phys 2000;47:365-71.

16. Heijkoop ST, Westerveld GH, Bijker N, et al. Optimal patient positioning (prone versus supine) for VMAT in gynecological cancer: a dosimetric study on the effect 
of different margins. Int J Radiat Oncol Biol Phys 2016;96:432-9.

17. Estabrook NC, Bartlett GK, Compton JJ, et al. Role of belly board device in the age of intensity modulated radiotherapy for pelvic irradiation. Med Dosim 2016;41:300-4.

18. Sawayanagi S, Yamashita H, Ogita M, et al. Volumetric and dosimetric comparison of organs at risk between the prone and supine positions in postoperative radiotherapy for prostate cancer. Radiat Oncol 2018;13:70.

19. Drzymala M, Hawkins MA, Henrys AJ, et al. The effect

Cite this article as: Yang Y, Cai S, Zhao T, Peng Q, Qian J, Tian Y. Effect of prone and supine treatment positions for postoperative treatment of rectal cancer on target dose coverage and small bowel sparing using intensity-modulated radiation therapy. Transl Cancer Res 2020;9(2):491-499. doi: 10.21037/ tcr.2019.11.33 of treatment position, prone or supine, on dose-volume histograms for pelvic radiotherapy in patients with rectal cancer. Br J Radiol 2009; 82:321-7.

20. Wiesendanger-Wittmer EM, Sijtsema NM, Mujis CT, et al. Systematic review of the role of a belly board device in radiotherapy delivery in patients with pelvic malignancies. Radiother Oncol 2012;102:325-34.

21. Stromberger C, Kom Y, Kawgan-Kagan M, et al. Intensity-modulated radiotherapy in patients with cervical cancer. An intra-individual comparison of prone and supine positioning. Radiat Oncol 2010;5:63-8. 